\title{
ANTICHOLINERGIC DRUGS - FUNCTIONAL ANTIDOTES FOR THE TREATMENT OF TABUN INTOXICATION
}

\author{
Gabriela Krejčová, Jiřı Kassa \\ Purkyně Military Medical Academy in Hradec Králové, Czech Republic: Department of Toxicology
}

\begin{abstract}
Summary: 1. To study the influence of antidotes on tabun-induced neurotoxicity, the rats were injected intramuscularly with organophosphate tabun $\left(\mathrm{LD}_{50}\right)$. The efficacy of choice antidotal treatment consisting of acetylcholinesterase reactivator obidoxime and one of four anticholinergic drugs (atropine, benactyzine, biperiden, scopolamine) was compared. 2. Testing of tabun-induced neurotoxicity progress was carried out using the method Functional observational battery. The experimental animals as well as controls were observed at 24 hours and 7 days following tabun or saline administration. 3 . The results were compared to the condition of animals without anticholinergic drug (oxime alone) and control rats that received physiological solution instead of tabun and treatment. Antidotal treatment involving centrally acting anticholinergic drugs (benactyzine, biperiden, scopolamine) showed significantly higher neuroprotective efficacy compared to antidotal treatment containing atropine.
\end{abstract}

Key words: Nerve agents; Tabun; Atropine; Benactyzine; Biperiden; Scopolamine; Functional observational battery

\section{Introduction}

The inhibition of acetylcholinesterase (AChE, EC 3.1.1.7) by organophosphorus compounds (OPs) causes acute toxicity or death of the intoxicated animals because of endogenic increase in acetylcholine (ACh) level in the cholinergic nervous system, that leads to muscle fasciculations, respiratory distress and epileptic fits. Consequential generalized seizures lead to severe incapacitation and to brain damage with lesions especially in hippocampus, piriform cortex $(12,16)$ and other cortical structures (8). The lesion is a highly selective distal axonopathy affecting the distal axons of sensory, motor, and autonomic neurons, and the longer central tracts. The lower motor neuron axonopathy is reversible, but not that of the upper motor neuron (17). Within the central nervous system, excitotoxicity has been described as a consequence of poisoning. Pathogenesis probably involves a combination of hypoxia and cholinergically mediated excitotoxicity with secondary recruitment of glutamatergic excitotoxicity (14). One group of these compounds, called nerve agents, pose an increasing threat in the world due to their possible use in the battlefield or terrorist acts. Therefore, inactivation of nerve agents has become a subject of major importance. Antidotes containing oxime compounds to reactivate the inhibited enzyme AChE are highly valued for the treatment of OP poisonings (7). Apart from oximes, the current antidotal treatment of nerve agent-induced acute poisoning in- cludes anticholinergic drugs to antagonize the effects of ACh excess at cholinergic receptor sites (15).

\section{Methods}

Male albino Wistar rats weighing $180-220 \mathrm{~g}$ were purchased from Konárovice (Czech Republic) and used in our experiment. The animals were kept in an air-conditioned room and allowed to access to standard food and tap water ad libitum. They were divided into groups of eight animals. Handling of the experimental animals was done under the supervision of the Special Committee of the Ministry of Defence according to $\S 23$ paragraph 1 a); the low of the Czech Republic no. 246/1992.

Tabun of $89.25 \%$ purity was obtained from Military Technical Institute in Brno (Czech Republic). Its purity was assayed by acidimetric titration. The oxime was synthesised at the Department of Toxicology of the Military Medical Academy and was $98 \%$ pure. Its purity was analysed using HPLC. All other chemicals and drugs of analytical grade were obtained commercially and used without further purification.

The anticholinergic drugs (atropine, benactyzine, biperiden, scopolamine) in combination with obidoxime were used as antidotal treatment and were carried out by i.m. injection 1 min following tabun administration at a lethal dose $\left(130 \mu \mathrm{g} / \mathrm{kg}\right.$ b.w. $\left.-\mathrm{LD}_{50}\right)$. The doses of obidoxime (4.2 $\mathrm{mg} / \mathrm{kg}$ b.w.) and anticholinergic drugs atropine $(25.2 \mathrm{mg} / \mathrm{kg}$ 
Tab. 1: Functional Observational Battery (FOB).

\begin{tabular}{|c|c|c|c|c|c|c|c|c|c|c|}
\hline \multirow{2}{*}{ MARKER } & \multicolumn{10}{|c|}{ Scored values only } \\
\hline & -2 & -1 & 0 & 1 & 2 & 3 & 4 & 5 & 6 & 7 \\
\hline POSTURE & & & & $\begin{array}{l}\text { sitting or } \\
\text { standing }\end{array}$ & rearing & asleep & flattened & $\begin{array}{l}\text { lying } \\
\text { on side }\end{array}$ & $\begin{array}{l}\text { crouched } \\
\text { over }\end{array}$ & $\begin{array}{c}\text { head } \\
\text { bobbing }\end{array}$ \\
\hline CATCH DIFFICULTY & & & & passive & normal & $\begin{array}{l}\text { elevated } \\
\text { activity }\end{array}$ & flight & escape & $\begin{array}{l}\text { aggrres- } \\
\text { sion }\end{array}$ & \\
\hline EASE OF HANDLING & & & & passive & normal & $\begin{array}{c}\text { moderately } \\
\text { difficult }\end{array}$ & difficult & & & \\
\hline MUSCULAR TONUS & atonia & hypotonia & normal & hypertonia & rigidity & $\begin{array}{c}\text { fascicula- } \\
\text { tions }\end{array}$ & & & & \\
\hline LACRIMATION & & & none & slight & severe & crusta & $\begin{array}{l}\text { coloured } \\
\text { crusta }\end{array}$ & & & \\
\hline PALPEBRAL CLOSURE & & & & open & $\begin{array}{l}\text { slightly } \\
\text { drooping }\end{array}$ & $\begin{array}{l}\text { half-way } \\
\text { drooping }\end{array}$ & $\begin{array}{c}\text { completely } \\
\text { shut }\end{array}$ & ptosis & & \\
\hline ENDO-EXOPHTHALMUS & & endo & normal & exo & & & & & & \\
\hline FUR ABNORMALITIES & & & normal & coloured & tousled & $\begin{array}{l}\text { color.+ } \\
\text { tousl. }\end{array}$ & blaze & injury & $\begin{array}{c}\text { other } \\
\text { changes }\end{array}$ & $\begin{array}{c}\text { pilo- } \\
\text { erection }\end{array}$ \\
\hline SKIN ABNORMALITIES & & & normal & pale & erythema & cyanosis & pigmented & cold & injury & \\
\hline SALIVATION & & & none & sllight & severe & & & & & \\
\hline NOSE SECRETION & & & none & slight & severe & coloured & & & & \\
\hline CLONIC MOVEMENTS & & & normal & $\begin{array}{c}\text { repetitive } \\
\text { movements } \\
\text { of mouth } \\
\text { and jaws }\end{array}$ & $\begin{array}{c}\text { non- } \\
\text { rhythmic } \\
\text { quivers }\end{array}$ & $\begin{array}{c}\text { mild } \\
\text { tremors }\end{array}$ & $\begin{array}{l}\text { severe } \\
\text { tremors }\end{array}$ & $\begin{array}{c}\text { myoclonic } \\
\text { jerks }\end{array}$ & $\begin{array}{c}\text { clonic } \\
\text { convulsions }\end{array}$ & $\begin{array}{c}\text { wet dog } \\
\text { shakes }\end{array}$ \\
\hline TONIC MOVEMENTS & & & normal & $\begin{array}{c}\text { contraction } \\
\text { of } \\
\text { extensors }\end{array}$ & $\begin{array}{c}\text { opistho- } \\
\text { tonus }\end{array}$ & $\begin{array}{c}\text { emprostho- } \\
\text { tonus }\end{array}$ & $\begin{array}{l}\text { explosive } \\
\text { jumps }\end{array}$ & $\begin{array}{c}\text { tonic } \\
\text { convul- } \\
\text { sions }\end{array}$ & & \\
\hline GAIT & & & normal & ataxia & $\begin{array}{c}\text { overcom- } \\
\text { pensation } \\
\text { of } \\
\text { hindlimbs } \\
\text { move- } \\
\text { ments }\end{array}$ & $\begin{array}{c}\text { feet point } \\
\text { outwards } \\
\text { from } \\
\text { body }\end{array}$ & $\begin{array}{l}\text { forelimbs } \\
\text { are } \\
\text { extended }\end{array}$ & $\begin{array}{l}\text { walks on } \\
\text { tiptoes }\end{array}$ & $\begin{array}{c}\text { hunched } \\
\text { body }\end{array}$ & $\begin{array}{l}\text { body is } \\
\text { flattened } \\
\text { against } \\
\text { surface }\end{array}$ \\
\hline GAIT SCORE & & & & normal & $\begin{array}{l}\text { slightly } \\
\text { impaired }\end{array}$ & $\begin{array}{c}\text { somewhat } \\
\text { impaired }\end{array}$ & $\begin{array}{l}\text { totally } \\
\text { impaired }\end{array}$ & & & \\
\hline MOBILITY SCORE & & & & normal & $\begin{array}{l}\text { slightly } \\
\text { impaired }\end{array}$ & $\begin{array}{c}\text { somewhat } \\
\text { impaired }\end{array}$ & $\begin{array}{c}\text { totally } \\
\text { impaired }\end{array}$ & & & \\
\hline $\begin{array}{l}\text { AROUSAL } \\
\text { (level of unprovoked activity) }\end{array}$ & & & & very low & sporadic & reduced & normal & enhanced & permanent & \\
\hline TENSION & & & none & $\begin{array}{l}\text { partial } \\
\text { (ears) }\end{array}$ & stupor & & & & & \\
\hline TENSION & & & none & $\begin{array}{c}\text { partial } \\
\text { (ears) }\end{array}$ & stupor & & & & & \\
\hline STEREOTYPY & & & none & $\begin{array}{c}\text { head } \\
\text { weaving }\end{array}$ & $\begin{array}{c}\text { body } \\
\text { weaving }\end{array}$ & grooming & circling & others & & \\
\hline BIZARRE BEHAVIOR & & & none & head & body & $\begin{array}{c}\text { self- } \\
\text { mutilation }\end{array}$ & $\begin{array}{c}\text { abnormal } \\
\text { movements }\end{array}$ & others & & \\
\hline APPROACH RESPONSE & & & & $\begin{array}{c}\text { no } \\
\text { reaction }\end{array}$ & normal & freeze & $\begin{array}{c}\text { energetic } \\
\text { reaction }\end{array}$ & $\begin{array}{c}\text { exaggerated } \\
\text { reaction }\end{array}$ & & \\
\hline TOUCH RESPONSE & & & & $\begin{array}{c}\text { no } \\
\text { reaction }\end{array}$ & normal & freeze & $\begin{array}{c}\text { energetic } \\
\text { reaction }\end{array}$ & $\begin{array}{c}\text { exaggerated } \\
\text { reaction }\end{array}$ & & \\
\hline CLICK RESPONSE & & & & $\begin{array}{c}\text { no } \\
\text { reaction }\end{array}$ & normal & freeze & $\begin{array}{c}\text { energetic } \\
\text { reaction }\end{array}$ & $\begin{array}{c}\text { exaggerated } \\
\text { reaction }\end{array}$ & & \\
\hline TAIL - PINCH RESPONSE & & & & $\begin{array}{c}\text { no reaction } \\
\text { reaction }\end{array}$ & normal & ffreeze & $\begin{array}{c}\text { energetic } \\
\text { reaction }\end{array}$ & $\begin{array}{c}\text { exaggerated } \\
\text { reaction }\end{array}$ & & \\
\hline PUPIL SIZE & & miosis & normal & mydriasis & & & & & & \\
\hline PUPIL SIZE & $\begin{array}{c}\text { miosis } \\
\text { consider- } \\
\text { able } \\
\end{array}$ & $\begin{array}{c}\text { miosis } \\
\text { slight }\end{array}$ & normal & $\begin{array}{c}\text { mydriasis } \\
\text { slight }\end{array}$ & $\begin{array}{c}\text { mydriasis } \\
\text { consider- } \\
\text { able }\end{array}$ & & & & & \\
\hline PUPIL RESPONSE & & & $\begin{array}{c}\text { no } \\
\text { reaction }\end{array}$ & $\begin{array}{l}\text { normal } \\
\text { reaction }\end{array}$ & & & & & & \\
\hline RIGHTING REFLEX & & & & normal & $\begin{array}{c}\text { slightly } \\
\text { uncoordin. }\end{array}$ & $\begin{array}{l}\text { lands on } \\
\text { side }\end{array}$ & $\begin{array}{c}\text { lands on } \\
\text { back }\end{array}$ & $\begin{array}{c}\text { rise from } \\
\text { back spon- } \\
\text { taneously }\end{array}$ & $\begin{array}{c}\text { rise from } \\
\text { back with } \\
\text { stimulus }\end{array}$ & $\begin{array}{c}\text { no } \\
\text { reaction }\end{array}$ \\
\hline
\end{tabular}


b.w.), benactyzine (3.7 mg/ $\mathrm{kg}$ b.w.), biperiden $(4.1 \mathrm{mg} / \mathrm{kg}$ b.w.) and scopolamine ( $15.2 \mathrm{mg} / \mathrm{kg} \mathrm{b.w.)}$ correspond to human-relevant doses $\left(2 \%\right.$ of their $\left.\mathrm{LD}_{50}\right)(1,5)$. The neurotoxicity of tabun was monitored using the Functional observational battery (FOB) at 24 hours and 7 days following tabun poisoning. The evaluated markers of tabun-induced neurotoxicity in experimental animals were compared to the parameters obtained from control rats, administered with saline instead of tabun and antidotes at the same volume $(0.1 \mathrm{ml} / 100 \mathrm{~g} \mathrm{~b}$. w. $)$.

The FOB consists of 40 measures of sensory, motor and autonomic functions (Tab. 1) $(3,9,10,11)$. First measurements were made while the animal was in the home cage. The observer evaluated each animal posture, palpebral closure, and presence or absence of convulsions. If convulsions were present, they were further categorized. Following observations in the home cage, the animal was removed and briefly held in the hand. Ease of removal and handling, skin and fur abnormalities, lacrimation, salivation and nose secretion were recorded.

Then, the rat was placed on a flat surface, which served as the open field covered with a clean absorbent pad. A timer was started for three minutes during which time the frequency of rearing responses was noted. At the same time, gait characteristics were noted and ranked, and arousal, tremor, convulsions and abnormal postures were evaluated. At the end of the time period ( $3 \mathrm{~min}$ ), the number of faecal boluses and urine pools on the absorbent pad were recorded. Reflex testing followed next and consisted of recording each rat's responses to the frontal approach of a blunt object such as a pencil, a touch of an object to the posterior flank, and an auditory click stimulus. Reactivity to a pinch on the tail and the ability of the pupil to constrict in response to light were also assessed. These measures were followed by a test for the righting reflex, then by measures of forelimb and hindlimb grip strength, body weight, rectal temperature and finally hindlimb foot splay. The entire battery of tests required approximately from six to eight minutes per one rat.

Motor activity data were collected shortly after FOB testing, using an apparatus for testing of a spontaneous motor activity of laboratory animals (constructed in Purkyne Military Medical Academy, Hradec Králové, Czech Republic). The animals were placed for a short time (10 minutes) in the measuring cage and their movements (total, horizontal and vertical activity) were recorded.

Data recorded with the FOB and motor activity assessment include categorical, ordinal and continuous values. Statistical analyses were performed on a PC with a special interactive programme NTX (3). The categorical and ordinal values were formulated as contingency tables and judged consecutively by Chi-squared test of homogeneity, Concordance-Discordance test and Kruskal-Wallis test respectively. The continual data were assessed by successive statistical tests: CI for Delta, Bartlett test for Equality of Variance, Williams test and Test for Distribution Function. The differences were considered significant when $\mathrm{p}<0.05$.

\section{Results}

The results obtained from testing at 24 hours or 7 days following tabun poisoning are shown in Table 2 and 3. One non-treated tabun-poisoned rat and one tabun-poisoned rat treated with obidoxime alone died within 24 hours following tabun administration. All fully treated tabun-poisoned rats survived till the end of experiment ( 7 days following the intoxication). The evaluation of tabun-induced neurotoxic signs at 24 hours following intoxication proved significant alteration of 23 observed parameters (Tab. 2). All tested possibilities of antidotal treatment of tabun-poisoned rats brought marked improvement in many of studied parameters. The results confirm that an addition of atropine to obidoxime does not cause to an increase in neuroprotective effect in comparison with obidoxime alone. On the other hand, if obidoxime is combined with the centrally acting anticholinergic drugs (benactyzine, biperiden, scopolamine), the tabun-poisoned rats were higher protected from acute neurotoxicity compared to obidoxime alone. The sitting posture of animal, low rearing and significant differences in landing foot splay, grip strength, body weight and body temperature were recorded at 7 days following tabun administration. Nevertheless, it is not possible to differenciate the efficacy of all combinations of antidotes of tabunpoisoned rats because of very few tabun-induced signs of neurotoxicity (Tab. 3).

\section{Discussion}

Tabun-induced toxic effects are extraordinarily difficult to counteract due to very low reactivating efficacy of currently used oximes $(2,4,13)$. The recent development of pharmacological protection from tabun-induced neurotoxicity focuses to revelation suitable prophylaxis and/or to current antidotal treatment. The anticholinergic drugs seem to be appropriate to enhance effectiveness of our protective possibilities. The previous published experiments $(5,6)$ established potency of the anticholinergics as prophylaxis. Nowadays, the commonly used anticholinergic drug for antidotal treatment is peripherally acting atropine. In our study, we examined the efficacy of centrally acting anticholinergics in a role of antidote. Our results suggest that all studied centrally acting anticholinergic drugs (benactyzine, biperiden, scopolamine) in the combination with oxime are able to increase the protection of animals against tabuninduced neurotoxicity. Their neuroprotective effect was more marked compared to atropine, because no improvement of improvement of therapeutic effect of antidotal treatment consisting of atropine and obidoxime was observed compared to obidoxime alone. Among centrally acting anticholinergics, biperiden showed the highest effect. In conclusion, the administration of centrally acting anticholinergic drugs in combination with the obidoxime appears to be the hopeful antidotal treatment of poisoning with nerve agents such as tabun. 


\begin{tabular}{|c|c|c|c|c|c|c|c|c|c|c|c|c|c|c|c|}
\hline \multicolumn{2}{|c|}{24 hours } & \multicolumn{2}{|c|}{ Controls } & \multicolumn{2}{|c|}{$\begin{array}{c}\text { Tabun + } \\
\text { Obidoxime + } \\
\text { Biperiden }\end{array}$} & \multicolumn{2}{|c|}{$\begin{array}{c}\text { Tabun }+ \\
\text { Obidoxime }+ \\
\text { Benactyzine }\end{array}$} & \multicolumn{2}{|c|}{$\begin{array}{c}\text { Tabun + } \\
\text { Obidoxime + } \\
\text { Atropine }\end{array}$} & \multicolumn{2}{|c|}{$\begin{array}{c}\text { Tabun }+ \\
\text { Obidoxime }+ \\
\text { Scopolamine }\end{array}$} & \multicolumn{2}{|c|}{$\begin{array}{c}\text { Tabun }+ \\
\text { Obidoxime }\end{array}$} & \multicolumn{2}{|c|}{ Tabun } \\
\hline No & Marker & $\mathrm{x} / \mathrm{m}$ & $\pm \mathrm{s}$ & $\mathrm{x} / \mathrm{m}$ & $\pm \mathrm{s}$ & $\mathrm{x} / \mathrm{m}$ & $\pm s$ & $\mathrm{x} / \mathrm{m}$ & $\pm \mathrm{s}$ & $\mathrm{x} / \mathrm{m}$ & $\pm \mathrm{s}$ & $\mathrm{x} / \mathrm{m}$ & $\pm \mathrm{s}$ & $\mathrm{x} / \mathrm{m}$ & $\pm \mathrm{s}$ \\
\hline 1 & posture & 1,63 & & 1,50 & & 1,57 & & $2,75^{*}$ & & 1,00 & & 2,38 & & $3,25^{*}$ & \\
\hline 2 & catch difficulty & 2,00 & & 1,63 & & $1,14^{* * * *}$ & & $1,50 * * *$ & & $1,43^{*}$ & & $1,63^{*}$ & & $1,63 * * *$ & \\
\hline 3 & ease of handling & 2,00 & & 1,63 & & $1,14^{* * * *}$ & & $1,25^{* * * *}$ & & $1,43^{*}$ & & $1,57 * *$ & & $1,75^{* *}$ & \\
\hline 4 & muscular tonus & 0,00 & & $-0,38$ & & $-0,86^{* * *}$ & & $-0,88^{* * *}$ & & $-0,43$ & & $-0,63$ & & $-1,13^{* * *}$ & \\
\hline 5 & lacrimation & 0,00 & & 0,00 & & 0,00 & & 0,00 & & 0,00 & & 1,00 & & $1,50^{*}$ & \\
\hline 6 & palpebral closure & 1,00 & & 1,00 & & 1,00 & & 1,00 & & 1,00 & & 1,50 & & 1,50 & \\
\hline 7 & endo-exophthalmus & 0,00 & & 0,14 & & 0,14 & & 0,25 & & 0,33 & & 0,25 & & $1,00^{* * * *}$ & \\
\hline 8 & fur abnormalities & 0,00 & & 0,00 & & 0,00 & & 0,38 & & 0,00 & & 0,88 & & 0,88 & \\
\hline 9 & skin abnormalities & 0,00 & & 0,00 & & 0,00 & & 0,25 & & 0,43 & & 0,00 & & 0,00 & \\
\hline 10 & salivation & 0,00 & & 0,13 & & 0,00 & & 0,14 & & 0,00 & & 0,63 & & $0,75 * * *$ & \\
\hline 11 & nose secretion & 0,00 & & 0,00 & & 0,00 & & $1,88^{* * *}$ & & 0,00 & & 0,75 & & $1,75^{* *}$ & \\
\hline 12 & rearing & 18,00 & 5,86 & 15,38 & 29,68 & $7,86^{* * * *}$ & 6,04 & $7,43^{* * *}$ & 5,26 & $3,00 * * *$ & 3,37 & 13,57 & 10,37 & $1,88^{* * *}$ & 1,36 \\
\hline 13 & urination & 0,25 & & 0,63 & & 0,57 & & 0,00 & & 2,00 & & 0,00 & & 0,38 & \\
\hline 14 & $\begin{array}{l}\text { defecation } \\
\end{array}$ & 0,00 & & 0,13 & & 0,38 & & 0,00 & & 0,63 & & 0,25 & & 0,00 & \\
\hline 15 & tremor & 0,00 & & 1,88 & & 1,29 & & 1,88 & & 1,14 & & 1,38 & & 2,50 & \\
\hline 16 & clonic movements & 0,00 & & 0,25 & & 0,14 & & 0,00 & & 0,00 & & 0,38 & & 0,38 & \\
\hline 16 & tonic movements & 0,00 & & 0,38 & & 0,00 & & 0,38 & & 0,13 & & 1,00 & & 2,50 & \\
\hline 17 & gait & 0,00 & & $0,75^{*}$ & & $0,86^{* *}$ & & $1,13 * * *$ & & 0,29 & & 1,38 & & $1,75^{* * *}$ & \\
\hline 18 & gait score & 1,00 & & $1,88^{* * * *}$ & & $2,00^{* * * *}$ & & $2,00 * * *$ & & $1,43^{*}$ & & $1,88^{*}$ & & $2,63^{* * *}$ & \\
\hline 20 & ataxia & 0,00 & & 0,25 & & $0,75^{*}$ & & $0,86^{* * * *}$ & & 0,29 & & 0,50 & & $1,13^{* * * *}$ & \\
\hline 19 & mobility score & 1,00 & & 1,25 & & 1,25 & & $1,63 * *$ & & 1,13 & & 1,63 & & $2,38^{* * *}$ & \\
\hline 20 & arousal & 4,00 & & 3,75 & & 3,50 & & 3,63 & & 3,63 & & 3,75 & & 2,75 & \\
\hline 21 & tension & 0,00 & & 0,25 & & 0,00 & & 0,00 & & 0,00 & & 0,25 & & 0,25 & \\
\hline 22 & stereotypy & 0,00 & & 0,00 & & 0,50 & & 0,00 & & 0,00 & & 0,50 & & 0,00 & \\
\hline 23 & bizzare behavior & 0,00 & & 0,00 & & 0,00 & & 0,00 & & 0,00 & & 0,00 & & 0,00 & \\
\hline 24 & approach response & 2,25 & & 2,00 & & 1,88 & & 2,00 & & 1,88 & & 2,25 & & 2,38 & \\
\hline 25 & touch response & 2,00 & & 2,00 & & 1,80 & & 2,00 & & 2,10 & & 1,80 & & 1,80 & \\
\hline 26 & $\begin{array}{l}\text { click response } \\
\end{array}$ & 2,00 & & 2,00 & & 2,13 & & 2,00 & & 2,25 & & 1,88 & & 2,25 & \\
\hline 27 & tail-pinch response & 2,00 & & $1,50^{*}$ & & 2,00 & & 1,75 & & 1,75 & & $1,50^{*}$ & & $1,25^{* * *}$ & \\
\hline 28 & pupil size & 0,00 & & $-0,50$ & & $-0,13$ & & $-0,25$ & & $-0,38$ & & $-0,50$ & & 0,00 & \\
\hline 29 & pupil response & 1,00 & & 1,00 & & 1,00 & & 1,00 & & 0,88 & & 0,69 & & 0,50 & \\
\hline 30 & righting reflex & 1,00 & & 1,00 & & 1,00 & & 1,13 & & 1,13 & & 2,13 & & 2,00 & \\
\hline 31 & landing foot splay $(\mathrm{mm})$ & 94,13 & 20,14 & 103,31 & 26,75 & 86,71 & 13,05 & $69,75^{*}$ & 12,66 & 96,79 & 21,19 & $58,13^{*}$ & 32,67 & $58,44 * *$ & 30,34 \\
\hline 32 & forelimb grip strength $(\mathrm{kg})$ & 3,53 & 1,44 & 2,94 & 0,97 & 2,67 & 0,43 & 2,74 & 1,09 & 2,47 & 0,36 & $2,08^{*}$ & 1,17 & $2,08^{*}$ & 1,11 \\
\hline 33 & hindlimb grip strength $(\mathrm{kg})$ & 0,96 & 0,43 & 0,97 & 0,43 & 0,92 & 0,12 & 0,63 & 0,43 & 0,81 & 0,14 & 1,18 & 1,44 & $0,48^{*}$ & 0,35 \\
\hline 34 & grip strength of all limbs $(\mathrm{kg})$ & 7,64 & 1,46 & 8,22 & 3,58 & 7,24 & 0,97 & $5,76^{*}$ & 1,11 & 6,76 & 0,83 & $5,28^{*}$ & 2,63 & $5,22^{*}$ & 2,36 \\
\hline 35 & food receiving (\%) & 100,00 & 0,00 & 100,00 & 0,00 & 100,00 & 0,00 & 100,00 & 0,00 & 100,00 & 0,00 & 50,00 & 0,00 & 50,00 & 0,00 \\
\hline 36 & body weight $(\mathrm{g})$ & 237,25 & 13,35 & 220,86 & 20,65 & $212,14 * *$ & 12,29 & $219,13^{*}$ & 11,81 & $202,43^{* *}$ & 16,43 & $209,14 *$ & 17,98 & 181,88 & 73,64 \\
\hline 37 & body temperature $(\mathrm{oC})$ & 37,16 & 0,39 & 37,14 & 0,30 & 37,27 & 0,29 & $36,60^{*}$ & 0,34 & 37,13 & 0,23 & 37,33 & 0,44 & $36,44^{*}$ & 0,57 \\
\hline 38 & vertical activity ( $\mathrm{No} / 10 \mathrm{~min})$. & 95,86 & 24,86 & $60,50 * * *$ & 35,34 & $43,86^{* * *}$ & 31,40 & $0,88^{* * * *}$ & 0,83 & $22,86^{* * *}$ & 44,93 & $29,13^{* * *}$ & 24,87 & $8,17 * * *$ & 13,38 \\
\hline 39 & horizontal activity ( $\mathrm{No} / 10 \mathrm{~min}$.) & 332,43 & 80,59 & $168,57^{* * *}$ & 88,68 & $178,14^{* * *}$ & 108,40 & $43,75^{* * *}$ & 23,37 & $120,71^{* * * *}$ & 120,94 & $176,50^{* *}$ & 146,86 & $66,17 * * *$ & 52,07 \\
\hline \multirow[t]{2}{*}{40} & total motor activity $(\mathrm{No} / 10 \mathrm{~min})$. & 428,29 & 165,69 & $229,07 * * *$ & 101,07 & 222,00 & 135,96 & $44,63^{* * * *}$ & 22,65 & $143,57^{* * *}$ & 163,76 & 205,63 & 170,62 & $74,34 * * *$ & 63,96 \\
\hline & & \multicolumn{2}{|c|}{$\mathrm{n}=8$} & \multicolumn{2}{|c|}{$\mathrm{n}=8$} & \multicolumn{2}{|c|}{$\mathrm{n}=8$} & $\mathrm{n}=8$ & & \multicolumn{2}{|c|}{$\mathrm{n}=8$} & \multicolumn{2}{|c|}{$\mathrm{n}=7$} & \multicolumn{2}{|l|}{$\mathrm{n}=7$} \\
\hline
\end{tabular}


Tab. 3

\begin{tabular}{|c|c|c|c|c|c|c|c|c|c|c|c|c|c|c|c|}
\hline \multicolumn{2}{|c|}{7 days } & \multicolumn{2}{|c|}{ Controls } & \multicolumn{2}{|c|}{\begin{tabular}{c|} 
Tabun + \\
Obidoxime + \\
Biperiden \\
\end{tabular}} & \multicolumn{2}{|c|}{$\begin{array}{c}\text { Tabun }+ \\
\text { Obidoxime }+ \\
\text { Benactyzine } \\
\end{array}$} & \multicolumn{2}{|c|}{$\begin{array}{c}\text { Tabun }+ \\
\text { Obidoxime }+ \\
\text { Atropine } \\
\end{array}$} & \multicolumn{2}{|c|}{$\begin{array}{c}\text { Tabun }+ \\
\text { Obidoxime + } \\
\text { Scopolamine }\end{array}$} & \multicolumn{2}{|c|}{$\begin{array}{c}\text { Tabun }+ \\
\text { Obidoxime }\end{array}$} & \multicolumn{2}{|c|}{ Tabun } \\
\hline No & Marker & $\mathrm{x} / \mathrm{m}$ & $\pm \mathrm{s}$ & $\mathrm{x} / \mathrm{m}$ & $\pm \mathrm{s}$ & $\mathrm{x} / \mathrm{m}$ & $\pm \mathrm{s}$ & $\mathrm{x} / \mathrm{m}$ & $\pm \mathrm{s}$ & $\mathrm{x} / \mathrm{m}$ & $\pm \mathrm{s}$ & $\mathrm{x} / \mathrm{m}$ & $\pm \mathrm{s}$ & $\mathrm{x} / \mathrm{m}$ & $\pm \mathrm{s}$ \\
\hline 1 & posture & 1,88 & & $1,38^{*}$ & & $1,25 * * *$ & & 1,63 & & $1,25 * * *$ & & $2,38^{*}$ & & $2,38^{*}$ & \\
\hline 2 & catch difficulty & 2,00 & & 1,71 & & 2,00 & & 2,57 & & 1,86 & & 2,00 & & 1,86 & \\
\hline 3 & ease of handling & 2,00 & & 1,75 & & 2,00 & & 1,75 & & 1,88 & & 2,00 & & 1,88 & \\
\hline 4 & muscular tonus & 0,00 & & 0,00 & & 0,00 & & 0,25 & & $-0,13$ & & \begin{tabular}{|l|l|}
$-0,38$ \\
\end{tabular} & & $-0,25$ & \\
\hline 5 & lacrimation & 0,00 & & 0,00 & & 0,00 & & 0,00 & & 0,00 & & 0,50 & & 0,50 & \\
\hline 6 & palpebral closure & 1,00 & & 1,00 & & 1,00 & & 1,00 & & 1,00 & & 1,50 & & 1,50 & \\
\hline 7 & endo-exophthalmus & 0,00 & & 0,00 & & 0,00 & & 0,00 & & 0,13 & & 0,13 & & 0,13 & \\
\hline 8 & fur abnormalities & 0,00 & & 0,00 & & 0,00 & & 0,00 & & 0,00 & & 1,13 & & 0,88 & \\
\hline 9 & skin abnormalities & 0,00 & & 0,00 & & 0,00 & & 0,00 & & 0,00 & & 0,38 & & 0,00 & \\
\hline 10 & salivation & 0,00 & & 0,00 & & 0,00 & & 0,00 & & 0,00 & & 0,25 & & 0,29 & \\
\hline 11 & nose secretion & 0,00 & & 0,00 & & 0,00 & & 0,00 & & 0,00 & & 0,38 & & 0,38 & \\
\hline 12 & rearing & 11,25 & 5,28 & 7,38 & 7,03 & 6,86 & 5,24 & $1,88^{* * * *}$ & 2,70 & $4,38^{*}$ & 4,10 & $3,83^{* *}$ & 5,19 & 7,13 & 6,85 \\
\hline 13 & urination & 0,38 & & 0,00 & & 1,71 & & 0,00 & & 0,00 & & 0,14 & & 0,14 & \\
\hline 14 & defecation & 0,38 & & 0,88 & & 0,29 & & 0,00 & & 0,00 & & 0,00 & & 1,14 & \\
\hline 15 & tremor & 0,00 & & 0,00 & & 0,00 & & 0,38 & & 0,00 & & 0,63 & & 0,71 & \\
\hline 16 & clonic movements & 0,00 & & 0,00 & & 0,00 & & 0,00 & & 0,13 & & 0,25 & & 0,25 & \\
\hline 16 & tonic movements & 0,00 & & 0,25 & & 0,00 & & 0,13 & & 0,13 & & 0,63 & & 0,86 & \\
\hline 17 & gait & 0,00 & & 0,75 & & 0,25 & & 0,75 & & 0,38 & & 1,38 & & 1,14 & \\
\hline 18 & \begin{tabular}{|l|} 
gait score \\
\end{tabular} & 0,00 & & 1,50 & & 1,38 & & 1,75 & & 1,13 & & 1,63 & & 1,86 & \\
\hline 20 & ataxia & 0,00 & & 0,25 & & 0,25 & & 0,63 & & 0,00 & & 0,50 & & 0,43 & \\
\hline 19 & mobility score & 1,00 & & 1,25 & & 1,25 & & 1,13 & & 1,13 & & 1,38 & & 1,43 & \\
\hline 20 & \begin{tabular}{|l|} 
arousal \\
\end{tabular} & 3,88 & & 3,00 & & 3,38 & & 3,13 & & 3,50 & & 2,75 & & 3,71 & \\
\hline 21 & tension & 0,00 & & 0,13 & & 0,13 & & 0,13 & & 0,13 & & 0,25 & & 0,71 & \\
\hline 22 & stereotypy & 0,00 & & 0,00 & & 0,00 & & 0,00 & & 0,00 & & 0,00 & & 0,00 & \\
\hline 23 & bizzare behavior & 0,00 & & 0,00 & & 0,00 & & 0,00 & & 0,00 & & 0,00 & & 0,00 & \\
\hline 24 & approach response & 1,75 & & 2,00 & & 2,00 & & 2,00 & & 1,88 & & 1,88 & & 1,86 & \\
\hline 25 & touch response & 1,50 & & 2,00 & & 2,00 & & 2,00 & & 1,88 & & 1,88 & & 1,86 & \\
\hline 26 & click response & 2,13 & & 2,00 & & 1,63 & & 2,25 & & 1,88 & & 2,00 & & 2,29 & \\
\hline 27 & tail-pinch response & 2,00 & & 2,00 & & 1,88 & & 2,00 & & 2,25 & & 1,63 & & 1,71 & \\
\hline 28 & pupil size & 0,00 & & 0,00 & & $-25,00$ & & $-0,38$ & & $-0,13$ & & $-0,25$ & & $-0,29$ & \\
\hline 29 & pupil response & 1,00 & & 1,00 & & 1,00 & & 0,88 & & 1,00 & & $\begin{array}{ll}0,88 \\
\end{array}$ & & 0,86 & \\
\hline 30 & righting reflex & 1,00 & & 1,00 & & 1,13 & & 1,25 & & 1,00 & & 1,75 & & 1,86 & \\
\hline 31 & landing foot splay $(\mathrm{mm})$ & 108,63 & 13,52 & 93,63 & 22,56 & 88,69 & 22,94 & 87,56 & 25,70 & $81,94^{*}$ & 16,94 & $74,86^{*}$ & 35,52 & $73,00^{*}$ & 37,29 \\
\hline 32 & forelimb grip strength $(\mathrm{kg})$ & 3,56 & 0,49 & 3,13 & 0,63 & $3,05^{*}$ & 0,37 & 3,06 & 0,71 & $2,67^{*}$ & 0,58 & $2,73^{*}$ & 0,41 & $2,42^{*}$ & 0,61 \\
\hline 33 & hindlimb grip strength $(\mathrm{kg})$ & 0,76 & 0,32 & $1,16^{*}$ & 0,18 & $1,54^{*}$ & 0,90 & $1,13^{*}$ & 0,32 & 1,06 & 0,22 & 1,08 & 0,36 & 0,80 & 0,29 \\
\hline 34 & grip strength of all limbs $(\mathrm{kg})$ & 8,78 & 0,60 & 8,46 & 1,77 & 8,98 & 1,32 & $7,52^{*}$ & 1,17 & 8,66 & 1,11 & 7,67 & 1,21 & 7,75 & 1,33 \\
\hline 35 & food receiving $(\%)$ & 100,00 & 0,00 & 100,00 & 0,00 & 100,00 & 0,00 & 100,00 & 0,00 & 100,00 & 0,00 & 100,00 & 1,00 & 100,00 & 0,00 \\
\hline 36 & body weight $(\mathrm{g})$ & 262,50 & 14,78 & $245,50^{*}$ & 16,23 & $242,50 *$ & 10,54 & 253,63 & 13,14 & $239,25^{*}$ & 18,70 & 238,67 & 32,30 & $246,43^{*}$ & 8,56 \\
\hline 37 & body temperature $(\mathrm{oC})$ & 37,21 & 0,44 & 37,65 & 0,58 & $37,83^{*}$ & 0,62 & $36,6^{* *}$ & 0,34 & $37,81^{*}$ & 0,55 & 37,68 & 0,44 & 37,80 & 0,29 \\
\hline 38 & vertical activity $(\mathrm{No} / 10 \mathrm{~min})$. & 33,63 & 26,25 & 60,00 & 50,52 & 48,86 & 34,552 & 20,71 & 22,79 & 53,50 & 79,24 & 13,33 & 12,06 & 10,29 & 14,66 \\
\hline 39 & horizontal activity $(\mathrm{No} / 10 \mathrm{~min})$. & 168,50 & 101,55 & 229,00 & 87,80 & 217,29 & 123,39 & 158,71 & 123,77 & 204,50 & 157,52 & 125,17 & 114,58 & $74,14^{*}$ & 49,49 \\
\hline \multirow[t]{2}{*}{40} & total motor activity $(\mathrm{No} / 10 \mathrm{~min})$. & 202,13 & 125,25 & 289,00 & 132,80 & 266,15 & 170,63 & 179,42 & 148,67 & 258,00 & 222,47 & 138,50 & 123,54 & 84,43 & 64,79 \\
\hline & & \multicolumn{2}{|c|}{$\mathrm{n}=8$} & \multicolumn{2}{|c|}{$\mathrm{n}=8$} & \multicolumn{2}{|c|}{$\mathrm{n}=8$} & \multicolumn{2}{|c|}{$\mathrm{n}=8$} & \multicolumn{2}{|c|}{$\mathrm{n}=8$} & \multicolumn{2}{|l|}{$\mathrm{n}=7$} & \multicolumn{2}{|c|}{$\mathrm{n}=7$} \\
\hline
\end{tabular}




\section{References}

1. Bajgar J, Fusek J, Vachek J. Treatment and prophylaxis against nerve agent poisoning. ASA Newslett. 1994;94:10-7.

2. Clement JG, Shiloff JD, Gennings C. Efficacy of a combination of acetylcholinesterase reactivators, $\mathrm{HI}-6$ and obidoxime, against tabun and soman poisoning in mice. Arch Toxicol 1987;61:70-5.

3. Frantík E, Hornychová M. Clustering of neurobehavioral measures of toxicity Homeostasis 1995;36:19-25.

4. Jokanovic M, Maksimovic M, Kilibarda V, Jovanovic D, Savic D. Oxime-induced reactivation of acetylcholinesterase inhibited by phosphoramidates. Toxicol Lett 1996;85:35-9.

5. Kassa J, Vachek J. A comparison of the efficacy of pyridostigmine alone and the combination of pyridostigmine with anticholinergic drugs as pharmacological pretreatment of tabun-poisoned rats and mice. Toxicology 2002;177:179-85.

6. Krejcova G, Kassa J. Neuroprotective efficacy of pharmacological pretreatment and antidotal treatment in tabun-poisoned rats. Toxicology 2003;185:129-39.

7. Luo Ch, Leader H, Radic $\mathrm{Z}$ et al. Two possible orientation of the HI-6 molecule in the reactivation of organophosphate-inhibited acetylcholinesterase. Biochem Pharmacol 2003;66(3):387-92.

8. McLeod CG, Singer W, Harrington DG. Acute neuropathology in soman poisoned rats. Fundam Appl Toxicol 1984;5:53-8.

9. Moser VC, Becking GC, Cuomo V et al. The IPCS collaborative study on neurobehavioral screening methods: III. Results of proficiency studies. Neurotoxicology 1997;18:939-46.

10. Moser VC, Becking GC, Cuomo V et al. The IPCS collaborative study on neurobehavioral screening methods: V. Results of chemical testing. Neurotoxicology 1997;18:969-1056
11. Moser VC, Tilson H, McPhail RC et al. The IPCS collaborative study on neurobehavioral screening methods: II. Protocol design and testing procedures. Neurotoxicology 1997;18:929-38.

12. Petras JM. Soman neurotoxicity. Fundam Appl Toxicol 1983;1:73-83.

13. Puu G, Artursson, E, Bucht G. Reactivation of nerve agent inhibited acetylcholinesterases by HI-6 and obidoxime. Biochem Pharmacol 1986;35:1505-10.

14. Solberg Y, Belkin M. The role of excitotoxicity in organophosphorus nerve agents central poisoning. Trends Pharmacol Sci 1997;18:183-5.

15. Taylor P. Anticholinesterase agents. In: Hardman JG, Limbird LE, editors. The Pharmacological Basis of Therapeutics, $9^{\text {th }}$ ed. New York: McGraw Hill, 1996 p.161-76.

16. Tonduli LS, Testylier G, Masqueliez C, Lallement G, Monmaur P. Effects of huperzine used as pre-treatment against soman-induced seizures. Neurotoxicology 2001;22(1):29-37.

17. Vasilesceu C, Floresceu C. Clinical and electrophysiological study of neuropathy after organophosphorus compound poisoning. Arch Toxicol 1980;43:305-15.

\section{Submitted October 2003.}

Accepted December 2003.

Mgr. Gabriela Krejčová,

Purkyně Military Medical Academy, 50001 Hradec Králové, Czech Republic. e-mail: krejcova@pmfhk.cz 